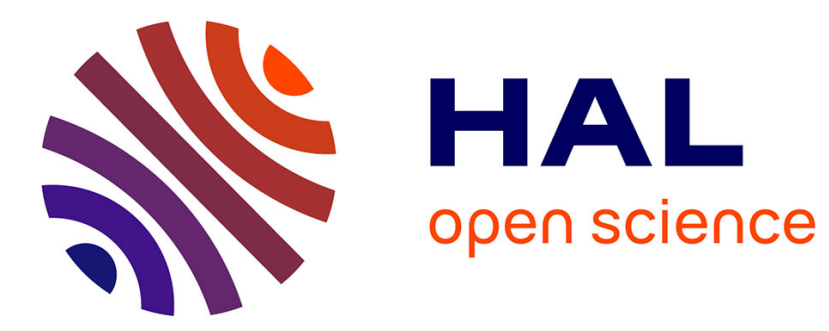

\title{
Chiral Pion interaction in nuclear matter and chiral symmetry restoration
}

\author{
G. Chanfray, D. Davesne
}

\section{To cite this version:}

G. Chanfray, D. Davesne. Chiral Pion interaction in nuclear matter and chiral symmetry restoration. Nuclear Physics A, 1999, 647, pp.125-131. 10.1016/S0375-9474(98)00615-0 . in2p3-00009565

\section{HAL Id: in2p3-00009565 https://hal.in2p3.fr/in2p3-00009565}

Submitted on 26 Jan 2001

HAL is a multi-disciplinary open access archive for the deposit and dissemination of scientific research documents, whether they are published or not. The documents may come from teaching and research institutions in France or abroad, or from public or private research centers.
L'archive ouverte pluridisciplinaire HAL, est destinée au dépôt et à la diffusion de documents scientifiques de niveau recherche, publiés ou non, émanant des établissements d'enseignement et de recherche français ou étrangers, des laboratoires publics ou privés. 


\title{
Chiral symmetry restoration of QCD and the Gross-Neveu model
}

\author{
T. Reisz*a

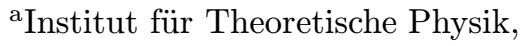 \\ Universität Heidelberg, \\ Philosophenweg 16, \\ D-69120 Heidelberg, Germany
}

Two flavour massless QCD has a second order chiral transition which has been argued to belong to the universality class of the $3 d \mathrm{O}(4)$ spin model. The arguments have been questioned recently, and the transition was claimed to be mean field behaved. We discuss this issue at the example of the $3 d$ Gross-Neveu model. A solution is obtained by applying various well established analytical methods.

\section{CHIRAL QCD}

Massless QCD has a flavour singlet, chiral symmetry that is broken spontaneously, but becomes restored at sufficently high temperature. For two flavours the symmetry restoring transition is widely accepted to be of second order. According to arguments first given by Pisarski and Wilczek, the universality class belongs to that of the $O(4)$ spin model in $3 \mathrm{~d}$. Beyond others, two main assumptions made are

- It is the symmetry pattern that determines the universality class.

- Dimensional reduction is complete at the phase transition, that is, quantum fluctuations along the temperature torus do not renormalize the classical $3 \mathrm{~d}$ IR behaviour.

In particular, the second assumption implies that fermions both do not interact as dynamical degrees of freedom in the IR, and do not even renormalize the (purely bosonic) effective theory in such a way that the universality class of the chiral transition changes.

Recently, the $O(4)$ scenario has been questioned, and the chiral transition was proposed to be mean field behaved.

*Supported by a Heisenberg fellowship, e-mail address t.reisz@thphys.uni-heidelberg.de

\section{THE GROSS-NEVEU MODEL}

The reliability of the arguments against or in favour of the classical reduction picture is resolved analytically in the $3 d$ Gross-Neveu (GN) model at finite temperature [1]. Here we outline the methods and the results.

The GN model we discuss is a four-point interacting model of $N$ two-component massless fermions, with partition function and action

$$
\begin{aligned}
& Z=\int \mathcal{D} \bar{\psi} \mathcal{D} \psi \exp \left(-S_{g n}(\bar{\psi}, \psi)\right) \\
& S_{g n}(\bar{\psi}, \psi)=\int_{x}\left(\bar{\psi} \gamma \cdot \partial \psi-\frac{\lambda^{2}}{N}(\bar{\psi} \psi)^{2}\right)(x)
\end{aligned}
$$

where $\int_{x} \equiv \int_{0}^{T^{-1}} d x_{0} \int_{\mathbf{R}^{2}} d^{2} \vec{x}$. We choose hermitian $\gamma_{i}$ in the representation with $\gamma_{0} \gamma_{1} \gamma_{2}=i \mathbf{1}_{2}$.

The model (1) serves as a good example for studying a "chiral" transition because it reveals considerable similarities to massless QCD. In $3 d$ the role of chirality is replaced by parity, corresponding to the transformation

$\psi(x) \rightarrow \psi(-x), \quad \bar{\psi}(x) \rightarrow-\bar{\psi}(-x)$.

The model is invariant under (2). The symmetry would be broken by a fermionic mass term $m \bar{\psi} \psi$. For sufficiently large coupling $\lambda$, parity symmetry is broken spontaneously at $T=0$, and it becomes restored at high temperature by a second order phase transition. The order parameter is given 
by

$\frac{1}{N}<\bar{\psi}(x) \psi(x)>$

which now represents the parity condensate.

For large $N$ it is convenient to rewrite the model as a Yukawa-type interacting model, using

$$
\begin{aligned}
& \exp \left(\frac{\lambda^{2}}{N}(\bar{\psi} \psi)^{2}\right)=\left(\frac{N}{4 \pi \lambda^{2}}\right)^{\frac{1}{2}} \\
& \int_{-\infty}^{\infty} d \sigma \exp \left(-\frac{1}{2}\left(\frac{N}{2 \lambda^{2}}\right) \sigma^{2} \pm \sigma \bar{\psi} \psi\right)
\end{aligned}
$$

Doing the quadratic fermionic integration, we obtain

$$
\begin{aligned}
& Z=\mathrm{c} \int \mathcal{D} \sigma \exp \left(-N S_{Y}(\sigma)\right) \\
& S_{Y}(\sigma)=\int_{z}\left(\frac{\sigma(z)^{2}}{4 \lambda^{2}}-\operatorname{tr}_{s} \log K(z, z)\right)
\end{aligned}
$$

with $K=\gamma \cdot \partial+\sigma \mathbf{1}$, and the trace is taken over the two spinor indices. We impose periodic boundary conditions on the auxiliary field $\sigma$ in temperature direction.

In the form (4) the GN model is studied by means of the saddle point expansion for large $N$. Whereas the expansion in powers of $\lambda$ is nonrenormalizable, the model becomes renormalizable in the $1 / N$-expansion. Renormalizability provides a large freedom in the choice of the UV cutoff. For simplicity, we choose a simple momentum cutoff, which respects parity symmetry.

\section{PHASE TRANSITION}

The minimizing configurations $\sigma(x)_{\min } \equiv \mu$ of the effective action (4) are obtained as the stable solutions of the gap equation

$\mu\left(\frac{1}{4 \lambda^{2}}-J_{1}\left(\mu^{2}, T^{-1}\right)\right)=0$,

with

$J_{1}\left(x, T^{-1}\right)=T \sum_{q_{0} \in \mathcal{F}} \int \frac{d^{2} \vec{q}}{(2 \pi)^{2}} \frac{1}{q^{2}+x}$,

where $\mathcal{F}=\{\pi T \nu \mid \nu \in \mathbf{Z}$ odd $\} . \mu(T)$ is equal to the parity condensate (3) to leading order in $1 / N$.
3.1. $\mathbf{N}=\infty$

At zero temperature, the broken phase is realized for sufficiently large coupling $\lambda$, and we set the $T=0$ mass scale $M$ by

$J_{1}\left(M^{2}, \infty\right) \equiv \frac{1}{4 \lambda^{2}}<J_{1}(0, \infty)$.

As function of $T, J_{1}$ is monotonically decreasing. The parity condensate $\mu(T)$ approaches zero continuously at $T_{c}=M /(2 \ln 2)$. It is straighforward to show that the critical exponents defined by the parity condensate and its correlation functions as $T \rightarrow T_{c}$ at $m=0$ (and $m \rightarrow 0$ at $T=T_{c}$ ) become $\gamma=1, \nu=1 / 2, \eta=0, \alpha=0, \beta=1 / 2($ and $\delta=3)$. These are the critical exponents as predicted by mean field analysis.

\subsection{FINITE N}

We solve the question of the universality class by applying the following two methods.

- Dimensional reduction. For large $N$ the phase transition is described by a $2 d$ scalar field theory. There is more than one universality class with the same symmetry. By computing the couplings of the effective model, we identify that part of the phase diagram that corresponds to the GN model.

- Linked cluster expansion. High temperature series expansions convergent up to the critical points are applied to the correlation functions of the effective model with the prescribed values of the coupling constants. The high order behaviour allows for a precise measurement of the critical exponents.

Dimensional reduction. This well established technique applies to local, renormalizable quantum field theories in $D \geq 3$ dimensions [2]. For sufficiently high temperature $T \geq T_{0}$, and for spatial momenta $\vec{k}$ sufficiently small w.r.t. $T$, the correlation functions are given by those of a $D-1$ dimensional field theory at zero temperature, which itself is local and renormalizable.

$$
\begin{aligned}
& <\widetilde{\phi}\left(\vec{k}_{1}\right) ; \ldots ; \widetilde{\phi}\left(\vec{k}_{n}\right)> \\
& =<\widetilde{\phi}\left(\vec{k}_{1}\right) ; \ldots ; \widetilde{\phi}\left(\vec{k}_{n}\right)>_{e f f}+O\left(\frac{\vec{k}_{1}}{T}, \ldots, \frac{\vec{k}_{n}}{T}\right) .
\end{aligned}
$$


The most general action is obtained by applying a zero-momentum expansion to the non-static effective action,

$$
\begin{aligned}
& S_{\text {eff }}^{\text {loc }}\left(\phi_{s t}\right)=\mathcal{T}_{\phi T^{(3-D) / 2}, \vec{k} / T}^{n, m, f(n, m)} \\
& {\left[-\ln \int \mathcal{D} \phi_{n s} \exp \left(-S\left(T^{1 / 2} \phi_{s t}+\phi_{n s}\right)\right)\right]}
\end{aligned}
$$

(this provides the loophole in the no-go theorem of Landsman [3]), where $\mathcal{T}_{x, y}^{n, m, f}$ denotes the Taylor expansion w.r.t. $x$ and $y$ of order $n$ and $m$, respectively, constrained by

$f(n, m) \equiv(D-1)-\left(\frac{D-3}{2} n+m\right) \geq 0$.

For the GN model, $T_{0}(N=\infty)=0$. For sufficiently large $N$, dimensional reduction works down to the phase transition temperature $T_{c}$. We get with the large $N$ expansion

$$
\begin{aligned}
& S_{e f f}(\phi)=c(T) \int_{\vec{x}}\left(\frac{m_{0}^{2}}{2} \phi(\vec{x})^{2}+\frac{1}{2}(\partial \phi)(\vec{x})^{2}\right. \\
& \left.+T^{2}\left[\frac{c_{4}}{N} \phi(\vec{x})^{4}-\frac{c_{6}}{N^{2}} \phi(\vec{x})^{6}+\frac{c_{8}}{N^{3}} \phi(\vec{x})^{8}\right]\right) .
\end{aligned}
$$

where we have truncated terms of order $N^{-4}$. All couplings are positive. They are known to leading orders of the $1 / N$ expansion.

Linked cluster expansion (LCE). According to renormalizability, we are allowed to put the effective model on the lattice. In a form well known for lattice spin models, the action becomes in terms of the lattice fields

$$
\begin{aligned}
& \widehat{S}_{0}\left(\phi_{0}\right)=\sum_{\vec{x} \in \Lambda_{2}}\left(-(2 \kappa) \sum_{\mu=1,2} \phi_{0}(\vec{x}) \phi_{0}(\vec{x}+\widehat{\mu})\right. \\
& \left.+\left[\phi_{0}^{2}+\sum_{i=2}^{4} \lambda_{i}\left(\phi_{0}^{2}-1\right)^{i}\right](\vec{x})\right) .
\end{aligned}
$$

LCE provides convergent series expansions of connected correlations and susceptibilities in powers of $\kappa$. For fixed $\lambda_{i}$ we have $\kappa \simeq T^{-1 / 2}$. Critical exponents are obtained from the high order behaviour of the coefficients. For instance, if

$$
\chi_{2}=<\widetilde{\phi}_{0}(0) ; \widetilde{\phi}_{0}(0)>=\sum_{L \geq 0} a_{L}(2 \kappa)^{L},
$$

Table 1

The exponents $\gamma$ and $\nu$ of the GN model.

\begin{tabular}{ccc}
\hline $\mathrm{N}$ & $\gamma(N)$ & $2 \nu(N)$ \\
\hline 46.1 & $1.751(2)$ & $2.001(2)$ \\
24.3 & $1.752(3)$ & $2.001(4)$ \\
4.68 & $1.749(2)$ & $1.999(2)$ \\
\hline
\end{tabular}

a critical behaviour as $\chi_{2} \simeq\left(\kappa_{c}-\kappa\right)^{-\gamma}$ is obtained from

$\frac{a_{L}}{a_{L-1}}=\frac{1}{2 \kappa_{c}}\left(1+\frac{\gamma-1}{L}+o\left(L^{-1}\right)\right)$.

The coefficients are computed to the 20th order. Some results on the exponents for various $N$ are summarized in Table 1. As a result, the parity restoring transition of the GN model belongs to the universality class of the $2 d$ Ising model.

\section{SUMMARY}

For the Gross-Neveu model with large number $N$ of flavours, dimensional reduction works down to the phase transition. The effective $2 d$ model has a rich phase structure, including both Gaussian and Ising model behaviour. However the values of the coupling constants are determined by the reduction technique. As a result of the computation, the parity transition of the Gross-Neveu model corresponds to the Ising part of the phase diagram.

For QCD we know that dimensional reduction works at least down to $2 T_{c}([4,5]$ and references therein). Whether it works down to $T_{c}$ or not is an open question. There are additional convergence factors $1 / T$ compared to the Gross-Neveu model because of the higher dimension, so $N$ is no more required to be large. However, the renormalized gauge coupling constant becomes large by approaching $T_{c}$, so that perturbation theory normally used in computing the effective action becomes worse. Other computational techniques are required to realize the reduction below $2 T_{c}$.

\section{REFERENCES}

1. T. Reisz, "The Gross-Neveu Model and QCDs Chiral Phase Transition", in "Field Theoretical Tools for Polymer and Particle Physics", 
Lecture Notes in Physics, Vol. 508 (1998) 192 H. Meyer-Ortmanns and A. Klümper, eds. (Springer Verlag, Heidelberg), and e-print archive hep-lat 9712017.

2. T. Reisz, Z. Phys. C53(1992)169

3. N. P. Landsman, Nucl. Phys. B322(1989)498

4. P. Lacock and T. Reisz, Nucl. Phys. (Proc. Suppl.) B30(1993)307

5. S. Dutta and S. Gupta, hep-ph/9806034 